\title{
Sound-based detection and ranging system as example application of a rapid prototyping and low-cost technology for board-level electronic systems education
}

\author{
Stefano Di Pascoli, Gabriele Ciarpi, Sergio Saponara \\ Dip. Di Ingegneria dell'Informazione, Università di Pisa, via G. Caruso 16, 56122, Pisa, I \\ Stefano.dipascoli@unipi.it; gabriele.ciarpi@unipi.it; sergio.saponara@unipi.it
}

\begin{abstract}
The work presents a low-cost and rapid prototyping technology for printed circuits board (PCB) design. The PCB design flow and the prototyping technology are presented, which are tailored for educational applications in University course of board-level electronic systems. Some fabricated samples with the proposed technology of educational electronic systems, designed by bachelor-level student teams, e.g. a sound-based detection and ranging system, are presented. The achieved results show the feasibility of the proposed technique as rapid prototyping tool and its suitability for engineering educational purpose.
\end{abstract}

\section{Introduction}

One of the main issue in Electronics educations is transferring the know-how in design, prototyping and testing of electronic systems, implemented using COTS (Commercial Off-the-Shelf) components at board-level, to students. Indeed, this subject of the Electronic field is still full of heuristics rules and usually require years of expertise to manage complex issues related to electrical, electromagnetic, mechanical and thermal aspects of components [1]. Moreover, a printed-circuit-board (PCB) electronic system design typically involves both analog and digital domains, power supply managements issues and low-power signal ones, and all non-ideal effects due to saturations, cross-talks, interference coupling, parasitic components. Multiple modeling approaches have to be used: e.g. both concentrated or distributed models since the signal frequency is continuously increasing due to the success of wireless systems and of high-speed digital ones. Finally, both CAD and prototyping technology concepts have to be transferred to the students considering the schematic design, components selection, functional, electrical and thermal simulations, thermal and reliability proper sizing, place and route design. The laboratory parts is also essential for board prototyping and assembling and testing. Due to the educational aim and to allow at home replicability of the experiments, then a free CAD flow and a simple technology have to be set up.

Several other students lab activities are employed in different tertiary level electronics courses [2-6]. However, actual system fabrication (PCB design and fabrication, components selection and procurement and final assembly and testing) is often overlooked and many lab activities concentrate in algorithm development $[3,4]$, or employ a commonly available or specially built platform $[2,6]$. 
To overcome these issues, this work presents a low-cost CAD flow and rapid prototyping technology for PCB design, tailored for educational applications in University course in Sections 2 and 3. A sample circuit designed by a bachelor-level student team and fabricated with the proposed technology, a sound-based detection and ranging system, is presented in Section 4. The achieved results are analyzed in Section 5 and show the feasibility of the proposed technique as rapid prototyping tool and its suitability for engineering educational purpose. Conclusions are drawn in Section 5.

\section{Board-level electronic systems education}

The Board Level Electronics (BLE) course (Italian: Costruzioni Elettroniche, CE) provides 6 ECTS units (60 hours of classroom lessons and supervised laboratory activities) and is focused on the applicative and industrial aspects of electronic system design. The enrolled students have a prerequisite curriculum, which includes the knowledge of signal theory, electronic devices theory, circuit theory and basic electronics. The course material can be divided into three main blocks:

1. "Circuit board theory": signal integrity, board parasitic effects and estimation, thermal aspects, reliability theory and estimation, basic rules for board level design. ( $50 \%$ of total time)

2. CAD tools for board development (15\%)

3. Supervised lab: The students are encouraged to divide into groups ( 1 to 4 people each) and to propose the development of an electronic system. (35\%)

The main purpose of the course is to present, for the first time, the students to the practical aspect of circuit fabrication. It is the first occasion for them to realize that circuit elements have a shape and a mass, that some solutions are easy and other are not, some are cheap and some are costly. In addition, the course aims to develop the teamwork skill of the students so as to introduce them in the industrial or research world, where the teamwork is the key to success. They are requested to use a high degree of autonomy to find and integrate multiple information about electrical, thermal, mechanical and electromagnetic aspects of components and their packages. They must develop a problem-solving attitude.

\section{Rapid prototyping and low-cost technology}

The proposed system is then developed using only free and/or open source software and low cost and easily available hardware, materials and tools.

Even if it is not an objective of the course, the participant students are encouraged to verify the functionality of the proposed system by means of a circuit simulator. Most popular free circuit simulators are Cadence ORCAD Pspice, lite edition and Linear LTSpice. The circuit simulation phase is of paramount importance for analog systems (or analog subsystems/frontends, in case of mixed-signal systems, like the circuit described further in this paper).

The rest of the development phases are to be developed with Kicad EDA suit. Kicad is reasonably complete and powerful, and has a large library of components and 
footprints. Only a few components and footprints, especially relative to electro mechanical components (switches, pushbuttons, connectors ...) cannot be found in Kicad libraries and have to be designed from scratch.

The participants use Kicad EEschema to design the schematic, select or design the components footprints, perform the annotation and electric rule check and generate the netlist. The PCB place and route phase is performed with Kicad Pcbnew, with a manual approach.

The chosen technology is based on the use of Surface Mount Devices (SMD) with a single copper top layer for interconnections. Electromechanical components (connectors, switches, etc...) are often mounted on the bottom face of the PCB, and soldered on the top layer, since this configuration provides much more mechanical strength and reliability than the use of SMD connectors

The students are instructed to use a minimum track width of $0.4 \mathrm{~mm}$, and a 0.3 clearance. These layout rules allow the use of components with a pin pitch of $0.8 \mathrm{~mm}$ like microcontrollers in TQFP package. Two-terminal components (resistors, capacitors, diodes, LED, ...) can use the 0805 SMD package.

The final PCB layout developed with the Kicad Pcbnew software is exported into the SWG file format. The tailored procedure used to move from an abstract SWG file to a real physic board is described in the next 6 stages:

1. The SWG file is printed with a common office laser printer (Epson AL-M200) on specially destrin-coated toner transfer paper.

2. This paper print is sandwiched, face down, on a $0.8 \mathrm{~mm}$ FR4 substrate coated with a $35 \mu \mathrm{m}$ copper layer and heated with a common office laminator. The heat glues the toner artwork to the copper surface.

3. After $5 \mathrm{~min}$ immersion in water the destrin coating releases the paper from the other side of the toner artwork and the paper can be removed. The toner layout now on copper is not able to withstand the copper chemical etching solution and has to be reinforced. This result can be obtained re-laminating the board with a polymer coated polyester foil (Toner Reactive Foil - TRF). A new layer of highopacity toner sticks to the original toner layer increasing its chemical robustness.

4. After the mask creation, the uncovered copper can be removed with a chemical attack. We use a mixture of tap water $(500 \mathrm{ml}) 38 \%-\mathrm{HCl}$ in water $(150 \mathrm{ml})$ and $30 \%$-hydrogen peroxide $(50 \mathrm{ml})$. The attack time is about 10 minutes. The final PCB is cleaned with acetone. Since we usually immediately solder the components, no copper finishing stage is used.

5. The holes for non-SMD components are manually performed with a vertical mount drill; the minimal hole diameter is $0.8 \mathrm{~mm}$. The maximum size of the boards that can be fabricated with the proposed technology are $210 \mathrm{~mm} \times 290 \mathrm{~mm}$, the limitation arises from the size of the destrin paper sheets used for the artwork printing and the maximum width of the office laminator The components are manually soldered with the help of a low magnification microscope. 


\section{Design examples: sound-based detection and ranging system}

Group 12 proposed a "sound based detection and ranging system": a system for the detection of the distance and direction (in two dimensions) of a loud sound. The system is composed by 3 omnidirectional microphones, three analog front ends based on an amplifier stage and filter, a comparator, a microcontroller (Atmega32) driving a LCD display, see Fig. 1

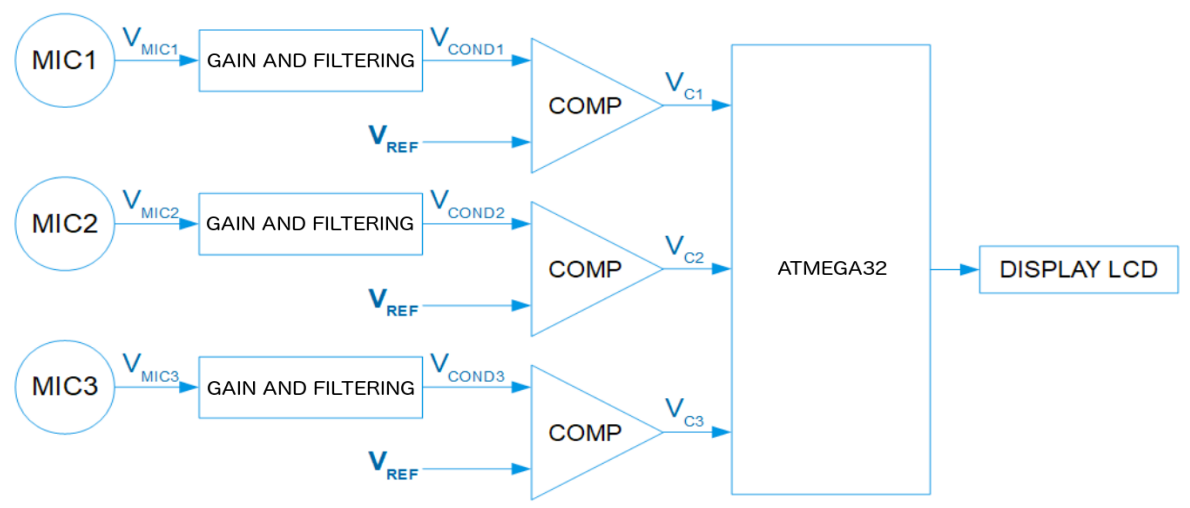

Fig. 1. Block diagram of the sound-based detection and ranging system.

The three fronts ends employ three OpAmp for a gain stage ( $30 \mathrm{~dB})$ and a second order Butterworth bandpass filter $(20 \mathrm{~Hz}-20 \mathrm{kHz})$. The amplified and band limited signal is than fed to a comparator with a user trimmable threshold. Finally, the three digital signals reach the interrupt input of the microcontroller, which implements the range and direction finding algorithm (implemented in $\mathrm{C}$ and assembly language) and drives an LCD display.

The circuit is powered at $5 \mathrm{~V}$ with a linear regulator and a $9 \mathrm{~V}$ battery.

The students provided also an activity record describing the development of the system. This report, besides the description of the circuits, contain a discussion of some power, thermal and reliability aspects of the system.

- A power consumption estimation of the circuit is provided together with a thermal discussion of the most critical component (the voltage regulator).

- Furthermore, the designers verified that their assumption of concentratedelements used during the design of the system was reasonable at the frequencies in use; they also assessed the effect of parasitic board effects (interconnection resistance, inductance and capacitance) and of thermal noise sources.

- Finally, a reliability analysis of the system has been performed, based on reliability data available for the microphone, the processor, the comparator and the OpAmp. 


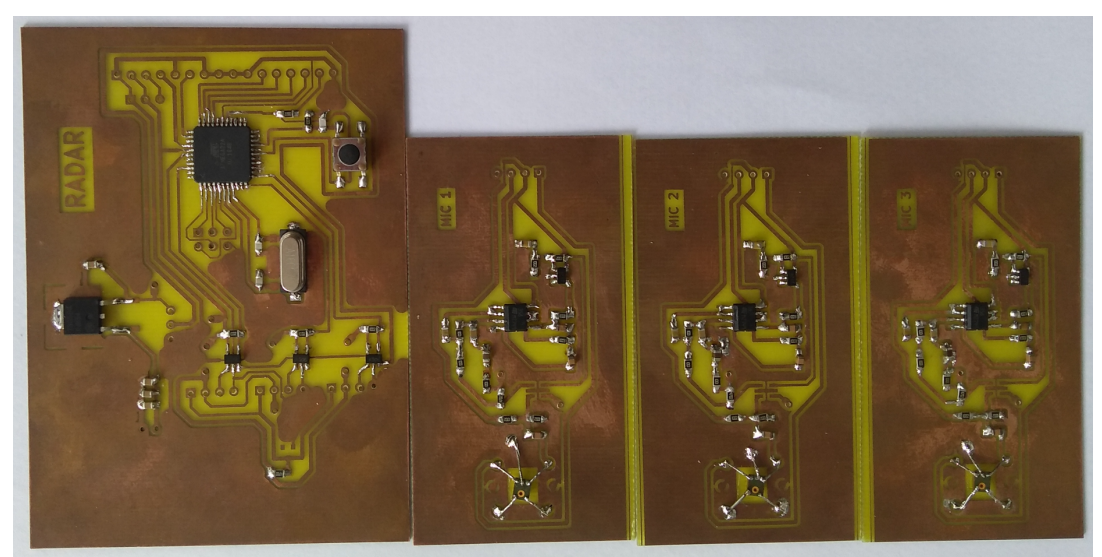

Fig. 2. Main board (top) and microphone boards (bottom 3) of the sound-based detection and ranging system

\section{Course statistics and conclusions}

Apart the specific example described in Section 4, Section 5 presents the course and project lab statistics. The projects are proposed and selected by the students, to stimulate their innovation attitude, but with guidance and suggestion from the teachers. The project development can be divided into three stages:

(1) Initial development: starting idea, analysis of the theory of operation, block diagram, gathering of information;

(2) Schematic development: selection of components and schematic design with Kicad Eeschema;

(3) PCB layout development with Kicad Pcbnew, PCB fabrication, assembling, and testing, using the technology described in Section 3. For projects using a microcontroller, this phase also includes firmware development.

Stages 1 and 2 were conducted in classroom with supervision. The selection of components was guided by means of the provision of a "suggested components list" (which included passive components, common discrete active devices, a few optical devices, a microprocessor, operational amplifiers, the 555 timer, some connectors and other commonly used devices). Other components could be acquired, with a cost constrain.

Stage 3 was performed partially as a homework (Kicad Pcbnew design) and partially with supervision (the fabrication process).

After the three stages are completed a technical documentation in English has to be produced, including a description of the 3 above stages at system, circuit and technology levels, and also including analysis of signal integrity, parasitic, thermal issues and proper cooling a/heatsink strategies, reliability and FMEA /Failure mode an defect analysis). The final exam is based on the analysis of the prototypes plus an oral session with $50 \%$ of the questions based on the implemented specific project plus $50 \%$ of questions related to theoretical aspects of CAD flow, design methodology or technology. Table 1 show the progress after about 6 weeks from the start of stage 3 . 
Table 1. Groups progress after about 6 weeks from the start of stage 3

\begin{tabular}{|c|l|l|}
\hline Group ID & Description & Status after 6 weeks \\
\hline 7 & Class D audio amplifier with spectral visualizer & Working \\
\hline 11 & Class AB audio amplifier & Working \\
\hline 2 & Psychedelic LED lights & Partially Working \\
\hline 8 & Rotating LED display & Working \\
\hline 9 & Greenhouse automator & Partially Working \\
\hline 6 & Drink dispenser & Working \\
\hline 4 & Two wheel robot & Testing \\
\hline 12 & Sound-based detection and ranging system & Testing \\
\hline 16 & Signal generator & Pcb ready \\
\hline 1 & FM sonar rangefinder & Pcb ready \\
\hline 3 & Robotic car with wrist remote & Pcb ready \\
\hline 10 & Theremin & Pcb development \\
\hline 13 & Magnetic gun & Pcb development \\
\hline 15 & Amplified loudspeaker & Pcb development \\
\hline
\end{tabular}

Acknowledgments. We thanks the members of the group 12, Marco Bellamio, Paolo Bonifati, Francesco Lombardi, Luca Lucarelli, who developed the describes system.

\section{References}

1.M. Awawdeh, T. Faisal, F. Fadhel and A. Al Hamadi, Improving electronics engineering students' skills by projects' college competition, 2018 Advances in Science and Engineering Technology International Conferences (ASET), Dubai, Sharjah, Abu Dhabi, United Arab Emirates, 2018, pp. 1-5.

doi: 10.1109/ICASET.2018.8376936

2. B. G. P. Cunha et al., DidacTronic: A low-cost and portable didactic lab for electronics: Kit for digital and analog electronic circuits, 2016 IEEE Global Humanitarian Technology Conference (GHTC), Seattle, WA, 2016, pp. 296-303.

doi: 10.1109/GHTC.2016.7857296

3. M. Portolan and R. Rolland, "Student-driven development of a digital tester," 2016 11th European Workshop on Microelectronics Education (EWME), Southampton, 2016, pp. 1-3. doi: 10.1109/EWME.2016.7496479

4.W. J. Esposito, F. A. Mujica, D. G. Garcia and G. T. A. Kovacs, "The Lab-In-A-Box project: An Arduino compatible signals and electronics teaching system," 2015 IEEE Signal Processing and Signal Processing Education Workshop (SP/SPE), Salt Lake City, UT, 2015, pp. 301-306. doi: 10.1109/DSP-SPE.2015.7369570

5.V. Nerguizian, R. Mhiri, M. Saad, H. Kane, J. S. Deschênes and H. Saliah-Hassane, "Lab@home for analog electronic circuit laboratory," 2012 6th IEEE International Conference on E-Learning in Industrial Electronics (ICELIE), Montreal, QC, 2012, pp. 110-115.

doi: 10.1109/ICELIE.2012.6471157

6. J. K. Lumpp, W. C. Blackburn, et al. "Instrumentation and measurement in a first-year engineering program", IEEE Instrumentation and Measurement Magazine, vol. 21, pp. 20-24, June 20187. https://www.orcad.com/resources/download-orcad-lite 